\title{
Structural and Functional Characteristics of Rat Hearts with and Without Myocardial Infarct. Initial Experience with Doppler Echocardiography
}

\author{
Valdir A. Moisés, Ricardo L. Ferreira, Emília N ozawa, Rosemeire M. Kanashiro, O rlando Campos Fo, \\ José Lázaro de Andrade, Antonio Carlos C. Carvalho, Paulo J. F. Tucci
}

São Paulo, SP - Brazil

\begin{abstract}
Objective - To assess by Doppler echocardiography the structural and functional alterations of rat heart with surgical induced extensive myocardial infarction.

Methods - Five weeks after surgical ligature of the left coronary artery, 38 Wistar-EPM rats of both sexes, 10 of them with extensive infarction, undergone anatomical and functional evaluation by Doppler echocardiography and then euthanized for anatomopathological analysis.
\end{abstract}

Results - Echocardiography was $100 \%$ sensible and specific to anatomopathological confirmed extensive miocardial infarction. Extensive infarction lead to dilatation of left ventricle (diastolic diameter: $0.89 \mathrm{~cm}$ vs. $0.64 \mathrm{~cm}$; systolic: $0.72 \mathrm{~cm} v s .0 .33 \mathrm{~cm}$ ) and left atrium $(0.55 \mathrm{~cm} v \mathrm{~s} .0 .33 \mathrm{~cm})$; thinning of left ventricular anterior wall (systolic: $0.14 \mathrm{~cm} v \mathrm{~s} .0 .23 \mathrm{~cm}$, diastolic: $0.11 \mathrm{~cm} v \mathrm{~s}$. $0.14 \mathrm{~cm}$ ); increased mitral $\mathrm{E} / \mathrm{A}$ wave relation (6.45 vs. 1.95). Signals of increased end diastolic ventricle pressure, B point in mitral valve tracing in $62.5 \%$ and signs of pulmonary hypertension straightening of pulmonary valve (90\%) and notching of pulmonary systolic flow $(60 \%)$ were observed in animals with extensive infarction.

Conclusion - Doppler echocardiography has a high sensitivity and specificity for detection of chronic extensive infarction. Extensive infarction caused dilatation of left cardiac chambers and showed in Doppler signals of increased end diastolic left ventricular pressure and pulmonary artery pressure.

Key words: myocardial infarct, rat hearts, Doppler echocardiogram

Universidade Federal de São Paulo - Escola Paulista de Medicina

Mailing address: Valdir A. Moisés - UNIFESP-EPM - Disciplina de Cardiologia

- Rua Botucatu, 740 - $2^{\circ}$ - 04023-900 - São Paulo, SP, Brazil

English version by Stela Maris C. Gandour
Myocardial infarct is one of the most frequent causes of heart failure and death in the human adult population ${ }^{1,2}$. Heart failure may be related to the extension of the infarct ${ }^{3,4}$. Clinical recognition of the myocardial infarct extension and of the degree of hemodynamic repercussion may be obtained through Doppler echocardiography ${ }^{5}$. Rat hearts with surgically induced myocardial infarcts have been analyzed with anatomicopathological studies, as well as with hemodynamic studies and, more recently, with Doppler echocardiography ${ }^{6-10}$. This latter diagnostic technique has been particularly interesting, mainly in serial studies and those with pharmacological interventions ${ }^{10,11}$. Because of our laboratory's interest in analyzing series of hearts of infarcted rats to test different therapeutical approaches, it became important to recognize the infarct and analyze its functional repercussions by Doppler echocardiography. Therefore this study aimed to report the main structural and functional findings by Doppler echocardiography of healthy rat hearts and rat hearts with extensive surgically induced myocardial infarct.

\section{Methods}

We selected 38 Wistar-EPM rats of both sexes that underwent echocardiography 5 weeks after undergoing surgical ligation of the left coronary artery. After that, the animals were euthanized for anatomicopathological study, which revealed no sign of myocardial infarct in 28 animals and the presence of myocardial infarct in 10 animals involving more than $40 \%$ of the left ventricular muscle. The 28 noninfarcted rats were 14 males and 14 females and all the 10 animals with extensive myocardial infarct were females. Their weights ranged from $240 \mathrm{~g}$ to $280 \mathrm{~g}$. All animals were kept inside plastic cages with air temperature ranging from $20^{\circ} \mathrm{C}$ to $22^{\circ} \mathrm{C}$, and light/dark cycles of 12 hours. They were fed with a Nunvilab food 
preparation and unrestricted water and received no medication before and after the procedure.

Myocardial infarct was induced according to a procedure previously described in the literature and adapted to our laboratory ${ }^{8}$. Briefly, the procedure consisted of the following: after anesthesia with $0.1 \mathrm{~mL} / \mathrm{kg}$ of a mixture of $0.67 \mathrm{mg} / \mathrm{kg}$ of xylazine and $0.33 \mathrm{mg} / \mathrm{mL}$ of ketamine administered through intraperitoneal via. The weight measurement was done on a Marte balance. The animals were placed in the dorsal decubitus position, immobilized, and underwent epilation and disinfection $\left(92.8^{\circ}\right.$ alcohol $)$ of the anterior facies of the thorax. Then, a surgical incision of $2 \mathrm{~cm}$ was performed in the skin along the left sternal margin, exposing the 5 th and 6 th ribs. After divulsion of the pectoralis and transversus muscles, a pouch suture was prepared to make possible the rapid closure of the thorax after surgery. Thoracotomy was then performed at the 5 th intercostal space for a rapid exteriorization of the heart by lateral compression of the thorax. The left coronary artery was then identified and occluded with a suture with a 5-0 polypropylene thread between the left atrial margin and the pulmonary artery. After a quick repositioning of the heart, the thorax was closed. To expand the thoracic cavity, pulmonary ventilation with a nasal mask was performed until the respiratory rate was stabilized.

Echocardiography of all animals was performed 5 weeks after infarct induction. For this, epilation of the anterior facies of the thorax was repeated as was sedation with the same solution used for surgery. The animals were then placed in the left lateral decubitus position $\left(45^{\circ}\right)$, and 3 electrodes were adhered to their paws for obtaining an electrocardiographic tracing simultaneously with the cardiac image that allowed identifying the phase of the cardiac cycle and recording of the respiratory rate. The examinations were performed with a commercially available device (Apogee Cx Ultrasound System - ATL Inc, Ambler, PA, USA) capable of producing images in the singledimensional or two-dimensional modes and of analyzing the intracardiac blood flow velocity with Doppler spectral analysis and color flow mapping techniques. A 7.5 MHz transducer was used at a depth of $3.0 \mathrm{~cm}$ and a sectorial angle of $60^{\circ}$. The length of the volume sample of the pulsed Doppler used was $0.6 \mathrm{~mm}$. Tracings of the M-mode, pulsed Doppler and simultaneous electrocardiogram were recorded at a velocity of $100 \mathrm{~mm} / \mathrm{s}$. At first, longitudinal images of the heart were obtained, including the left ventricle and atrium, the mitral valve, and the aorta, followed by the cross-sectional images from the plane of the base (aorta, left atrium, right ventricular outflow tract, and pulmonary valve and artery) to the left ventricular apical region. Concomitantly with obtaining of these images, M-mode tracings of the aortic root (including the aortic valve), the left atrium, the mitral and pulmonary valves, and the left ventricular cavity at the level of the papilary muscles were obtained. In the apical four-chamber view, we obtained the curve of velocity of the mitral diastolic flow, positioning the volume sample of the pulsed Doppler slightly below the ventricular facies of the mitral leaflets. Simultaneous recordings of the velocity curve of the flow of the left ventricular outflow tract and the mitral diastolic flow were obtained in the apical 4-chamber view, positioning the volume sample of the pulsed Doppler between the left ventricular outflow tract and inlet. We also analyzed the velocity curve of the systolic flow in the pulmonary artery in the cross-sectional parasternal view of the base with the volume sample of the pulsed Doppler positioned right below the valvar plane. Two-dimensional images and tracings of the M-mode and of the Doppler curves were recorded in half-inch videotapes for later analysis. Based on the left ventricular images of the cross-sectional (basal, middle, and apical levels) and longitudinal views, we defined myocardial infarct as hyperechoic or nonhyperechoic regions or segments of the myocardium showing one of the following changes in myocardial kinetics: hypokinesis (a reduction in wall thickness or motion), akinesis (no increase in wall thickness or no motion or both), and dyskinesis (opposite movement of one or more segments or regions).

The measurements derived from the M-mode tracings and from the flow velocity curves obtained by pulsed Doppler were performed with an off-line analysis system (Image-Vue, DCR 1.60, Nova Microsonics) in which the images recorded in videotapes were reproduced and digitalized in a sequence of 8 frames, the most appropriate image chosen for measurements. The following measures were obtained through M-mode echocardiography: left atrium at the end of systole, aortic root at the end of diastole, anterior and posterior wall diastolic thickness (AWDT, PWDT), anterior and posterior wall systolic thickness (AWST, PWST), left ventricular diastolic (Dd) and systolic (Sd) diameters, being the values expressed in centimeters $(\mathrm{cm})$. All measures were obtained following the recommendations of the American Society of Echocardiography ${ }^{12}$. Based on these measurements, left ventricular mass (in grams) was calculated according to the equation established by the American Society of Echocardiography $\mathrm{M}(\mathrm{g})=1.04$ [(Dd + AWDT + PWDT $\left.-\mathrm{Dd}^{3}\right]^{3}$ as was the percentage of left ventricular shortening (PLVS), PLVS (\%)= Dd-Sd/Dd x 100.

Because of segmentary myocardial impairment in infarcted animals, the myocardial mass and left ventricular shortening fraction were not estimated. These two parameters were calculated only with data of noninfarcted animals. Based on the curve of mitral diastolic flow, the maximum velocity of $\mathrm{E}$ and $\mathrm{A}$ waves was calculated in centimeters per second $(\mathrm{cm} / \mathrm{s})$ as was the ratio between these two waves (E/A ratio). The time of atrial deceleration expressed in milliseconds was estimated as the time between the maximum velocity of the $E$ wave in the curve of mitral diastolic flow and its intersection with the 
Doppler basal line. The left ventricular isovolumetric relaxation interval was estimated as the time ( $\mathrm{ms})$ between the end of the flow curve of the left ventricular outflow tract and the beginning of the velocity curve of the mitral diastolic flow. These parameters were used for analyzing left ventricular diastolic function.

After undergoing echocardiography, the animals were anesthetized with urethane at the dosage of $0.3 \mathrm{~mL} /$ $100 \mathrm{~g}$ of weight (solution of $400 \mathrm{mg} / \mathrm{mL}$ ) and underwent thoracotomy for heart removal. The real left ventricular weight was obtained in the analytical balance of Mettler after complete separation of the pericardium, right ventricle, atria, and great vessels. According to a technique reported by other authors ${ }^{8}$, an anatomicopathological study was carried out in these hearts to characterize the presence or absence of infarct. After fixation in a solution of $10 \%$ formol, 4 cross-sectional cuts in the left ventricle were performed. After discarding the cuts of the extremities, 3 cuts representing the basal, middle, and apical regions were stained with hematoxylin and eosin and Masson's trichrome for infarct search. When present, infarct size was estimated as the mean of the percentage of the scar arch length (or segmentary contractile change or both) in regard to the transversal circumference in the 3 views of the left ventricle ${ }^{8}$.

Echocardiographic variables were expressed by the median, maximum, and minimum values. The left ventricular mass obtained by echocardiography was compared to the values of real weight of the chamber using the Spearman correlation test. Values of the remaining variables studied in infarcted and noninfarcted animals were compared using the nonparametric Mann- Whitney test. Comparison of motion changes in mitral and pulmonary valves in the M-mode and the pattern of the curve of systolic pulmonary flow between animals with and without extensive myocardial infarct were performed through the test of comparison of proportions. Tests with $\mathrm{p}<0.05$ were considered statistically significant.

\section{Results}

Echocardiography provided images and Doppler signals adequate for the proposed analyses in all selected animals. Segmentary contractile changes in the left ventricle indicating infarct on echocardiogram were observed in 10 animals, and these findings were later confirmed as extensive myocardial infarcts on the anatomicopathological study. No animal considered noninfarcted on anatomicopathological study showed echocardiographic changes suggestive of left ventricular infarct. On echocardiogram, animals with extensive infarcts showed echodense areas in the left ventricular myocardium suggesting fibrosis or thinning of the myocardium or contractile change in the anterolateral and apical walls or both. Sometimes these areas extended to the inferior wall preserving

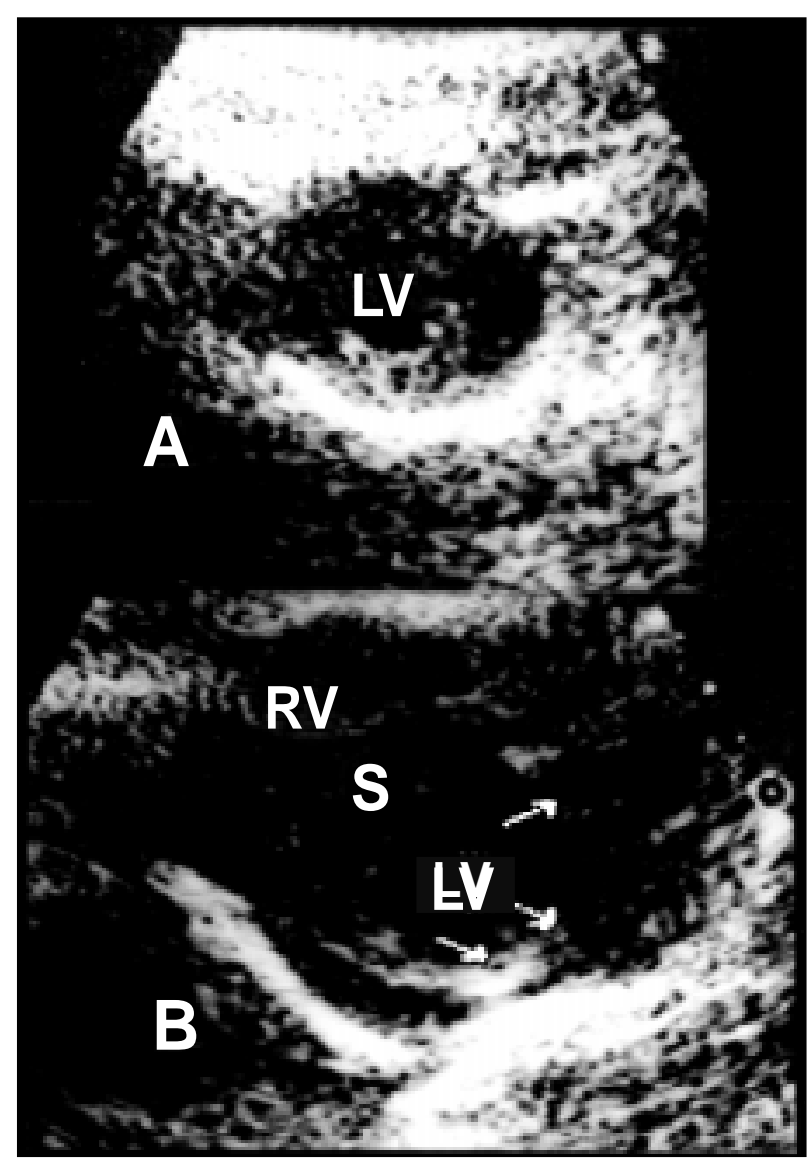

Fig. 1 - Images of the cross-sectional view of the left ventricle (LV) in a rat without infarct (A) and in another rat with extensive myocardial infarct (B). s- interventricular septum; arrows - myocardial infarct.

the interventricular septum, and these findings could be confirmed by the anatomicopathological study (fig. 1). Table I summarizes the maximum, minimum, and median values of left ventricular weight, heart rate during echocardiogram, and all structural variables of infarcted and noninfarcted animals. We may also observe the result of the statistical analysis of the comparison between these variables. Left ventricular mass of noninfarcted animals showed a significant correlation $(r=0.60 ; p=0.0092)$ with real left ventricular weight. Table II summarizes the maximum, minimum, and median values of functional variables of infarcted and noninfarcted animals. It is important to emphasize that E/A ratio values were significantly higher in animals with extensive infarcts than in those with no infarct (fig. 2). The mitral valve tracing on M-mode echocardiography showed a B point in $5(62.5 \%)$ out of 8 animals with extensive myocardial infarct and in no animal of the noninfarcted group ( $\mathrm{p}<0.05)$ (fig. 3$)$. The pulmonary valve tracing was straight (without A wave) in $9(90 \%)$ out of 10 animals with extensive infarct and in none of the animals of the noninfarcted group $(\mathrm{p}<0.05)$. Systolic flow in the pulmonary artery showed a mesosystolic notch in 6 $(60 \%)$ out of 10 infarcted animals and in none of the animals of the noninfarcted group $(\mathrm{p}<0.05)$ (fig. 4$)$. 


\begin{tabular}{|c|c|c|c|c|c|c|c|}
\hline \multirow[t]{2}{*}{ Variables } & \multicolumn{2}{|c|}{ Median } & \multicolumn{2}{|c|}{ Minimum } & \multicolumn{2}{|c|}{ Maximum } & \multirow[t]{2}{*}{$\mathrm{p}$} \\
\hline & Healthy & Infarcted & Healthy & Infarcted & Healthy & Infarcted & \\
\hline LV weight & 0.71 & 0.63 & 0.46 & 0.54 & 0.84 & 0.74 & 0.00 \\
\hline HR (bpm) & 263 & 230 & 180 & 214 & 416 & 260 & 0.34 \\
\hline $\operatorname{Ao}(\mathrm{cm})$ & 0.31 & 0.30 & 0.24 & 0.24 & 0.36 & 0.32 & 0.14 \\
\hline $\mathrm{LA}(\mathrm{cm})$ & 0.32 & 0.53 & 0.23 & 0.50 & 0.49 & 0.66 & 0.00 \\
\hline $\operatorname{Dd}(\mathrm{cm})$ & 0.64 & 0.88 & 0.53 & 0.81 & 0.73 & 1.0 & 0.00 \\
\hline $\operatorname{Ds}(\mathrm{cm})$ & 0.33 & 0.72 & 0.21 & 0.56 & 0.46 & 0.86 & 0.00 \\
\hline AWDT $(\mathrm{cm})$ & 0.14 & 0.11 & 0.10 & 0.08 & 0.19 & 0.16 & 0.001 \\
\hline AWST(cm) & 0.23 & 0.14 & 0.19 & 0.12 & 0.34 & 0.21 & 0.001 \\
\hline PWDT $(\mathrm{cm})$ & 0.15 & 0.12 & 0.12 & 0.10 & 0.36 & 0.20 & 0.05 \\
\hline PWST(cm) & 0.26 & 0.20 & 0.20 & 0.14 & 0.36 & 0.29 & 0.000 \\
\hline Mass (g) & 0.58 & $\longrightarrow$ & 0.40 & $\longrightarrow$ & 1.01 & - & \\
\hline
\end{tabular}

\begin{tabular}{|c|c|c|c|c|c|c|c|}
\hline \multirow[t]{2}{*}{ Variable } & \multicolumn{2}{|c|}{ Median } & \multicolumn{2}{|c|}{ Minimum } & \multicolumn{2}{|c|}{ Maximum } & \multirow[t]{2}{*}{$\mathrm{p}$} \\
\hline & Healthy & Infarcted & Healthy & Infarcted & Healthy & Infarcted & \\
\hline $\operatorname{LVSP}(\%)$ & 49.7 & -- & 33.3 & -- & 61.5 & -- & -- \\
\hline $\mathrm{E}(\mathrm{cm} / \mathrm{s})$ & 65.9 & 76.2 & 43.5 & 56.7 & 98.5 & 152 & 0.05 \\
\hline $\mathrm{A}(\mathrm{cm} / \mathrm{s})$ & 30 & 13.1 & 23 & 81 & 7.8 & 35.4 & 0.001 \\
\hline $\mathrm{E} / \mathrm{A}$ & 1.95 & 6.45 & 1.01 & 1.68 & 2.63 & 8.90 & 0.001 \\
\hline $\operatorname{MDD}(\mathrm{ms})$ & 40.1 & 41 & 33.4 & 23 & 61.9 & 45 & 0.48 \\
\hline LVIR (ms) & 23.8 & 27.6 & 16.5 & 10.4 & 41.8 & 38.3 & 0.62 \\
\hline \multicolumn{8}{|c|}{$\begin{array}{l}\text { A- velocity of the A wave in the curve of mitral diastolic flow }(\mathrm{cm} / \mathrm{s}) \text {; E- velocity of the E wave in the curve of diastolic mitral flow; E/A- ratio between the velocities } \\
\text { of E and A waves of the mitral diastolic flow; MDDT- mitral diastolic flow deceleration time (ms); LVIRI- left ventricular isovolumetric relaxation interval (ms); } \\
\text { LVSP- left ventricular shortening percentage }(\%) \text {. }\end{array}$} \\
\hline
\end{tabular}

\section{Discussion}

Myocardial infarct in rats has been used as a model for a few years. The great advantage of this model is the possibility of keeping the animals alive for long periods until healing of the infarcted myocardial area and appearance of signs of left ventricular remodeling occur. According to Spadaro et $\mathrm{al}^{8}$, left coronary artery ligature in the first epicardial segment, 3 to $4 \mathrm{~mm}$ after its origin, does not interrupt circulation to the septal branch, which in rats originates very close to the left coronary artery ostium. Therefore the interventricular septum may be kept intact in most infarcts caused by proximal occlusion of the left coronary artery in rats, while a great impairment of the left ventricular free wall is observed. It is worth remembering that after ligation of the left coronary artery, some animals develop extensive infarcts, while others develop smaller infarcts and others develop no infarct at all. In the present study using a similar technique, the anatomicopathological study of the heart revealed an extensive infarct involving the free wall of the cavity in 10 animals. The echocardiogram performed 5 weeks after the infarct and before the anatomicopathological study showed signs suggesting fibrosis and segmentary contractile change in the same left ventricular walls. Infarcted animals showed diastolic and systolic thickness of left ventricular anterior and posterior walls significantly smaller than those of noninfarcted animals. This may represent impairment of the myocardial walls involved by infarct and variable degrees of healing.

Five weeks later, all animals with myocardial infarct showed a significant increase in left cavity dimensions on Doppler echocardiogram. These findings were accompanied by important functional changes, such as signs of increase in the left ventricular diastolic pressure characterized by a restrictive type of the mitral diastolic flow (mainly the E/A ratio) in the velocity curve and the $\mathrm{B}$ point in the mitral valve tracing, both present only in extensively infarcted animals. The characteristics of the velocity cur- 


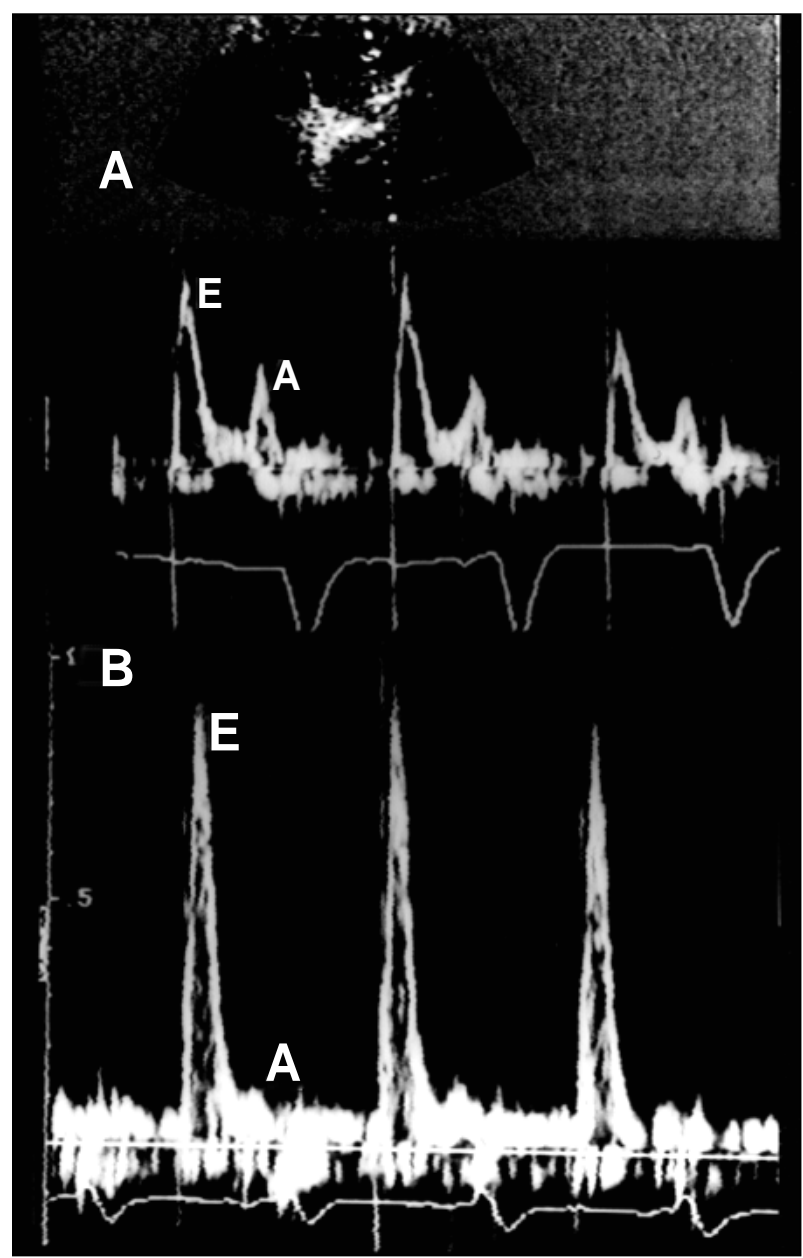

Fig. 2-Velocity curve of the mitral diastolic flow of a noninfarcted rat (A) and of another rat with an extensive myocardial infarct (B). Observe the increase in $E$ wave and the decrease in A wave in B.

ve of mitral diastolic flow suggesting a restrictive pattern of ventricular filling already reported in extensively infarcted rats ${ }^{10}$, as well as the finding of the $\mathrm{B}$ point in the mitral valve (M-mode), are similar to that observed in humans with significant heart failure due to myocardial infarct ${ }^{13}$. Findings of straightening of the pulmonary valve tracing on M-mode echocardiogram and the systolic notch in the velocity curve of the pulmonary flow have not yet been shown in rats with extensive myocardial infarct. Once again, the similarities between these findings and the pulmonary hypertension pattern in humans ${ }^{14}$ allow the supposition that they indicate an increase in the pressure of pulmonary circulation in rats with extensive infarct due to an increase in left ventricular diastolic pressure. In the present study, a hemodynamic assessment with direct measurements of the pulmonary arterial pressure and left ventricular diastolic pressure was not carried out for comparison. Indices of global left ventricular systolic function, such as the shortening percentage (or fraction) or ejection fraction or both, were analyzed only in noninfarcted animals. These methods use only left ventricular linear measurements for the calculus and do not represent

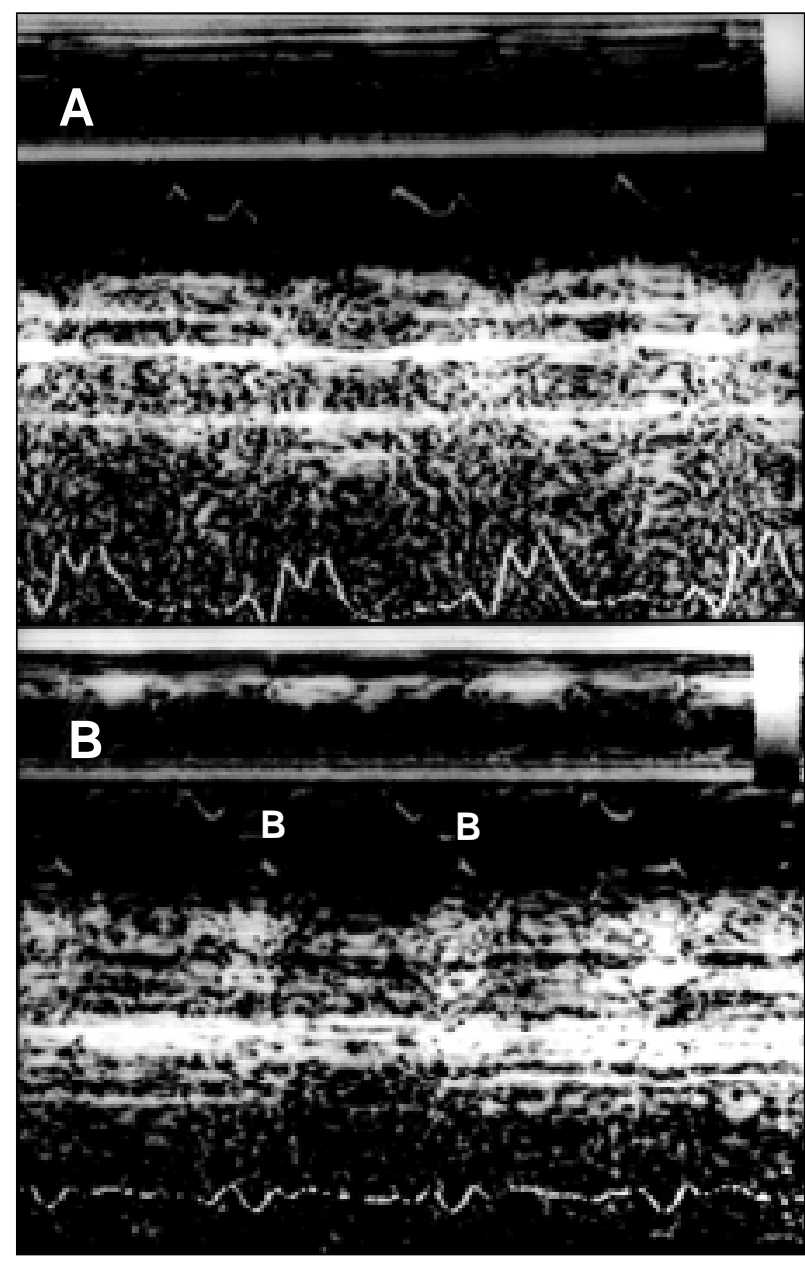

Fig. 3 - Tracings in M-mode of the mitral valve of a healthy rat (A) and of another rat with extensive myocardial infarct (B) with a B point in the enddiastolic phase.

the global systolic function in hearts with regional contractile changes, such as those in infarcted hearts. The two-dimensional methods traditionally used in humans are based on left ventricular images obtained in the apical view. However, the quality of the images in this view in the animals of the present study was not appropriate for obtaining reliable estimates of ejection fraction. A transducer with a higher frequency might provide a better definition of the endocardium in images obtained in the apical view ${ }^{15}$. Another alternative is the analysis of the percent variation of the left ventricular cross-sectional area because images in cross-sectional parasternal view are usually adequate. In the present study, this method was not used because we thought it would be interesting to test it in a group of animals with varied sizes of myocardial infarct to make comparison possible. An ongoing study in our laboratory will analyze this method in more detail.

Data regarding anatomic and functional variables of noninfarcted animals are important in this study because they allowed comparison between the variables of animals with extensive infarct and may be useful as reference 
values in future studies of our or other laboratories. The correlation index between left ventricular mass estimated by echocardiogram and the real weight of the cavity, although statistically significant, was low as compared with that reported by others ${ }^{16}$. One factor determining the low correlation index may have been the inclusion of animals with normal left ventricular mass and within a relatively narrow range of values. Including animals with greater body weight and others with left ventricular hypertrophy may widen the range of values and increase the correlation index, as has occurred in other studies. Animals with extensive infarct were not included because of the uneven variation in myocardial thickness of these hearts and because of variations in the geometrical shape of the cavity. It is worth noting that the frequency of the $7.5 \mathrm{MHz}$ transducer used, even though providing an appropriate image for identifying the structures analyzed, may have impaired detection of the real wall margins, mainly in the areas closer to the transducer. This may have been one reason for the low correlation index. Transducers with higher frequencies ( 10 or $12 \mathrm{MHz}$ ) may perhaps allow more accurate measurements of myocardial thickness and cavity diameters.

Despite some limitations and based on data and observations of the present study, we can conclude that Doppler echocardiography may identify extensive myocardial infarct with a 5-week evolution in rats. Infarcted animals showed an increase in the dimensions of the left cardiac cavities and indirect signs of an increase in left ventricular diastolic and pulmonary artery systolic pressures on Doppler echocardiography.

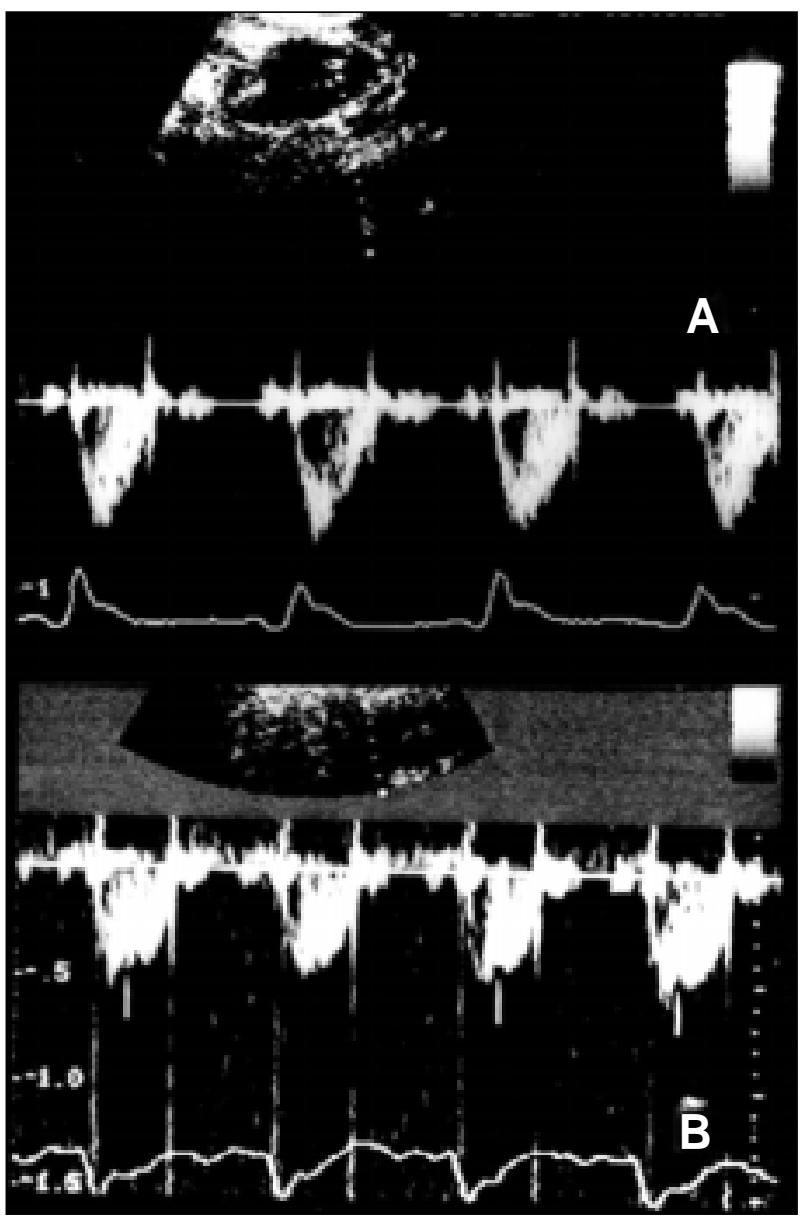

Fig. 4-Velocity curves of the systolic pulmonary flow in a rat with no infarct (A) and in another rat with extensive myocardial infarct (B) with mesosystolic notch (arrows).

\section{References}

1. Barreto ACP, Nobre MRC, Wajngarten M, Canesin MF, Ballas D, Serro-Azul JB. Insuficiência cardíaca em grande hospital terceário de São Paulo. Arq Bras Cardiol 1998; 71: 15-20.

2. Teerlink JR, Goldhaber SZ, Pfeffer MA. An overview of contemporary etiologies of congestive heart failure. Am Heart J 1991; 121: 1852-3.

3. Page DL, Caulfield JB, Kastor JA, DeSanctis RW, Sanders CA. Myocardial changes associated with cardiogenic shock. N Engl J Med 1971; 285: 133-7.

4. Nishimura RA, Reeder GS, MillerFA, et al. Prognostic value of predischarge 2-dimensional echocardiogram after acute myocardial infarction. Am J Cardiol 1984; 53: 429-32.

5. Nishimura RA, Tajik AJ, Shub C, Miller FA Jr, Ilstrup DM, Harrison CE. Role of two-dimensional echocardiography in prediction of in-hospital complications after acute myocardial infarction. J Am Coll Cardiol 1984; 4: 1080-7.

6. Litwin SE. The rat model of post-myocardial infarction heart failure. Heart Failure 1995; 2: 182-95.

7. PfefferMA, Pfeffer JM, Fishbein MC, FletcherPJ, Spadaro J, KlonerRA, etal. Myocardial infarct size and ventricular function in rats. Circ Res 1979; 44: 503-12.

8. Spadaro J, Fishbein MC, Hare C, Pfeffer MA, Maroko PR. Characterization of myocardial infarcts in the rat. Arch Pathol Lab Med 1980; 104: 179-83.

9. Baily RG, Lehman JC, Gubin SS, Musch TI. Noninvasive assessment of ventricular damage in rats with myocardial infarction. Cardiovasc Res 1993; 27: 851-5.
10. Litwin SE, Katz SE, Morgan JP, Douglas PS. Serial echocardiographic assessment of left ventricular geometry and function after large myocardial infarction in the rat. Circulation 1994; 89: 345-54.

11. Litwin SE, Katz SE, Morgan JP, Douglas PS. Long-term captopril treatment improves diastolic filling more than systolic performance in rats with large myocardial infarction. J Am Coll Cardiol 1996; 28: 773-81.

12. Sahn DJ, DeMaria A, Kisslo J, Weyman A. Recommendations regarding quantitation in M-mode echocardiography. Results of a survey of echocardiographic measurements. Circulation 1978; 58: 1072-83.

13. Oh JK, Ding ZP, Gersh BJ, Bailey KR, Tajik AJ. Restrictive left ventricular diastolic filling identifies patients with heart failure after acute myocardial infartcion. J Am Soc Echocardiogr 1992; 5: 497-503.

14. Campos $\mathrm{F}^{\circ} \mathrm{O}$, Andrade JL, Carvalho ACC, et al. Avaliação da pressão arterial pulmonar em adultos pela ecodopplercardiografia. Arq Bras Cardiol 1991; 56: 261-8.

15. Youn HJ, Rokosh G, Lester SJ, Simpson P, Schiller NB, Foster E. Two-dimensional echocardiography with a $15 \mathrm{MHz}$ transducer is a promising alternative for in vivo measurement of lef ventricular mass in mice. J Am Soc Echocardiogr 1999; 12: $70-5$.

16. Pawlush DG, Moore RL, Musch TL, Davidson Jr WR. Echocardiographic evaluation of size, function, and mass of normal and hypertrophied rat ventricles. $\mathrm{J}$ Appl Physiol 1993; 74: 2598-605. 